\title{
Estudio mediante difracción de Rayos-X de las tensiones residuales generadas por diferentes tipos de mecanizado
}

\author{
V. GARCÍA'1, N. ORDÁS', M.L. PENALVA², J. FERNÁNDEZ², K. OSTOLAZA³, C. GARCÍA-ROSALES'1 \\ ${ }^{1}$ Centro de Estudios e Investigaciones Técnicas de Guipúzcoa (CEIT) 20009 San Sebastián \\ ${ }^{2}$ Campus Tecnológico Universidad de Navarra (TECNUN) 20009 San Sebastián \\ ${ }^{3}$ Industria de Turbo Propulsores (ITP), S.A. 48170 Zamudio
}

\begin{abstract}
El propósito de este trabajo es la medición de tensiones residuales mediante difracción de rayos- $X$ en materiales sometidos a diferentes tipos de mecanizado, con el fin de mostrar la importancia que tiene una correcta elección del tipo y parámetros del mecanizado en la calidad de la superficie creada, especialmente en el estado final de tensiones residuales. Se han realizado mediciones sobre distintos materiales (acero inoxidable 17 / 7 PH, Ti-6Al-4V y acero de herramienta F-521), mecanizados mediante torneado duro o rectificado. En cada caso se ha obtenido la evolución del estado de tensiones con la distancia a la superficie. Se ha aplicado un método integral para deducir las tensiones residuales a partir de medidas de la variación en la posición del pico de difracción. Este método proporciona el tensor de tensiones completo, así como los tensores gradientes de tensiones en dirección longitudinal, transversal y en profundidad. Las medidas de difracción de rayos- $\mathrm{X}$ han permitido la evaluación de tensiones residuales tanto macroscópicas como microscópicas (éstas últimas solamente de forma cualitativa).
\end{abstract}

Palabras clave: Tensión Residual; Difracción de Rayos-X; Torneado; Rectificado

Evaluation of the residual stresses generated by different types of machining using $X$-ray diffraction.

The aim of the present work is the measurement of residual stresses using X-ray diffraction in materials subjected to different kinds of machining, to show the relevance of an adequate choice of machining type and parameters on the quality of the generated surface, especially in the residual stress final state. Different materials (stainless steel 17/7 PH, Ti-6Al-4V and F-521 tool steel) subjected to hard-turning and grinding have been investigated. In each case the evolution of the residual stress with distance to surface has been obtained. An integral method has been applied to determine the residual stress state by measuring the changes in the diffraction angle position. This method provides the full stress tensor and the stress gradient tensor in the longitudinal and transversal directions and in depth. X-ray diffraction measurements have allowed the evaluation of both macroscopic and microscopic residual stresses (the latter only qualitatively).

Keywords: Residual Stress; X-ray Diffraction; Turning; Grinding

\section{INTRODUCCIÓN}

Los principales problemas asociados con el mecanizado de aceros de herramienta están relacionados con la integridad de la superficie (14): tensiones residuales, martensita sin revenir (capa blanca, fase dura pero frágil), martensita sobrerevenida (fase blanda) y aparición de grietas. Durante los procesos de mecanizado la superficie de la pieza se ve afectada plástica, térmica y químicamente. La fuerza normal aplicada por la herramienta sobre la superficie de la pieza genera deformación plástica, dando lugar a tensiones residuales compresivas en la capa más superficial. La fricción entre la pieza y la herramienta es tal que genera temperaturas muy elevadas capaces de producir transformaciones de fase en las capas más superficiales del material y tensiones residuales tractivas $(1,5)$. Estas tensiones tractivas son perjudiciales para la vida del material durante su uso, puesto que favorecen la formación y propagación de grietas. Para conseguir un proceso de mecanizado satisfactorio, se requiere llegar a un compromiso entre estas dos tendencias opuestas; para ello es necesario controlar la generación de calor y conseguir que la superficie final se deforme plásticamente, para lo cual es preciso elegir unos parámetros de mecanizado adecuados.

Las tensiones residuales se clasifican en dos tipos: macroscópicas y microscópicas. Las macrotensiones se extienden a lo largo de distancias relativamente grandes, comparadas con el tamaño de grano del material, y se caracterizan mediante un tensor. Las microtensiones son aquéllas que se dan en torno a defectos y juntas de grano, tratándose en este caso de una propiedad escalar de la muestra.

En el presente trabajo se presentan las tensiones residuales generadas por torneado duro en acero de herramienta F-521 y por rectificado en acero inoxidable 17 / 7 PH y Ti-6Al-4V. Se ha obtenido la evolución en profundidad eliminando capas mediante electropulido, por lo que se ha realizado una corrección por eliminación de capas (6) para tener en cuenta la relajación de tensiones tras el electropulido.

\section{PARTE EXPERIMENTAL.}

\subsection{Materiales}

Se han estudiado tres muestras cilíndricas de acero de herramienta F-521 (AISI D2) (cuya composición nominal se presenta en la tabla 1.a): una sin mecanizar y las otras dos sometidas a torneado duro (una de ellas con herramienta nueva y la otra con herramienta gastada).

TABLA 1 COMPOSICIÓN NOMINAL (WT\%) DE LOS MATERIALES ESTUDIADOS: A) F-521 (AISI D2), B) ACERO INOXIDABLE 17/7PH, C) TI-6AL-4V

1.a

\begin{tabular}{||c|c|c|c|c|c|c|c|c|c|}
\hline $\mathrm{C}$ & $\mathrm{Mn}$ & $\mathrm{Si}$ & $\mathrm{Cr}$ & $\mathrm{Ni}$ & $\mathrm{Mo}$ & $\mathrm{V}^{*}$ & $\mathrm{Cu}^{*}$ & $\mathrm{P}^{*}$ & $\mathrm{~S}^{*}$ \\
\hline $1.4-1.6$ & 0.6 & 0.6 & $11.0-13.0$ & 0.3 & $0.7-1.2$ & 1.1 & 0.25 & 0.03 & 0.03 \\
\hline
\end{tabular}
${ }^{*}$ Composición máxima

1.b

\begin{tabular}{|c|c|c|c|c|c|c|c|}
\hline $\mathrm{Cr}$ & $\mathrm{Ni}$ & $\mathrm{Al}$ & $\mathrm{C}^{*}$ & $\mathrm{Mn}^{*}$ & $\mathrm{Si}^{*}$ & $\mathrm{P}^{*}$ & $\mathrm{~S}^{*}$ \\
\hline $16-18$ & $6.5-7.75$ & $0.75-1.5$ & 0.09 & 1 & 1 & 0.04 & $0.03-0.04$ \\
\hline
\end{tabular}
Composición máxima

1.c

\begin{tabular}{||c|c|c|c|c|c|c|}
\hline $\mathrm{Al}$ & $\mathrm{V}$ & $\mathrm{N}^{*}$ & $\mathrm{C}^{*}$ & $\mathrm{H}^{*}$ & $\mathrm{Fe}^{*}$ & $\mathrm{O}^{*}$ \\
\hline $5.5-6.75$ & $3.5-4.5$ & 0.05 & 0.1 & 0.0125 & 0.35 & 0.2 \\
\hline
\end{tabular}


Asimismo se han estudiado las tensiones residuales en varias probetas de fatiga de dos materiales de interés aeronáutico y en automoción (acero inoxidable 17/7 PH y Ti-6Al-4V, cuyas composiciones nominales se encuentran en las tablas 1 .b y 1 .c respectivamente) sometidos a dos procesos de rectificado diferentes.

\subsection{Electropulido y corrección por eliminación de capas.}

La evolución en profundidad de las tensiones residuales se ha obtenido eliminando sucesivas capas de material mediante pulido electrolítico. Las condiciones del proceso de electropulido para cada uno de los materiales se muestran en la tabla 2. A las tensiones obtenidas se les ha aplicado la corrección propuesta por Moore y Evans (6) para tener en cuenta la relajación de tensiones producida por la eliminación de capas. Como se esperaba, esta corrección es mínima, salvo para elevados espesores eliminados (del orden de $150 \mu \mathrm{m}$ y superiores) donde dicha corrección comienza a ser notable.

TABla 2. CONDICIONES DEL PROCESO DE PULIDO ELECTROLÍtiCO DE CADA UNO DE LOS MATERIALES ESTUDIADOS

\begin{tabular}{|l|l|l|l|}
\hline & F-521 & $17 / 7 \mathrm{PH}$ & Ti-6Al-4V \\
\hline \multirow{5}{*}{ Electrolito } & $70 \%$ Etanol & $70 \%$ Etanol & \\
& $12 \%$ Agua destilada & $12 \%$ Agua destilada & $94 \%$ Ácido Acético Glacial \\
& $10 \%$ 2-Butoxietanol & $10 \%$ 2-Butoxietanol & $6 \% \mathrm{HClO}_{4}$ \\
& $8 \% \mathrm{HClO}_{4}$ & \\
\hline Voltaje & $18 \mathrm{~V}$ & $29 \mathrm{~V}$ & $11-11.5 \mathrm{~V}$ \\
\hline
\end{tabular}

\subsection{Medidas de difracción de rayos- $X$ y análisis de datos.}

Las medidas de difracción de rayos-X se han llevado a cabo en un goniómetro horizontal Philips X'Pert MRD (PW3050/20 HR). Para los aceros F-521 y 17/ 7 PH se ha utilizado radiación $\mathrm{K}_{\alpha} \operatorname{de~} \mathrm{Cr}(\lambda=2.2897 \AA)$ mientras

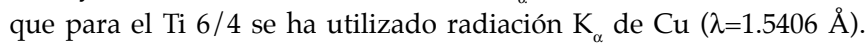
Los picos estudiados son: (211) de martensita (tetragonal centrada en el cuerpo) localizado a $2 \theta \approx 155.5^{\circ}$ en el acero F-521; (211) de ferrita (cúbica centrada en el cuerpo) localizado a $2 \theta \approx 154^{\circ}$ en el acero $17 / 7$ PH y (213) de la fase $\alpha$ (hexagonal) localizado a $2 \theta \approx 142^{\circ}$ en el Ti $6 / 4$. La geometría adoptada para las medidas es goniómetro- $\psi$. En todos los casos el espaciado interplanar se ha medido en trece inclinaciones $\psi \mathrm{y}$ tres orientaciones $\phi$ diferentes (es decir 39 orientaciones diferentes respecto de los ejes de la muestra). Las correcciones aplicadas a las intensidades medidas han sido: Lorentz-Polarización, fondo y desdoblamiento $\alpha_{2}$ (7). La posición de los picos se ha determinado utilizando ajuste a curva gausiana. La presencia de estados triaxiales de tensiones así como de gradientes de tensiones (caso del presente trabajo) imposibilita la aplicación del tradicional método del $\operatorname{sen}^{2} \psi$ y hace necesaria la aplicación de un método integral como el llamado método RIM, consistente en una integración ponderada (debido a la absorción, las capas superficiales contribuyen más a la amplitud de la señal) de la deformación normal a la superficie, expresada como una función polinómica del ángulo de inclinación $\psi$. Ésta matriz de deformaciones puede desarrollarse en serie de Taylor, introduciendo así los tensores gradientes de deformaciones en dirección longitudinal, transversal y en profundidad. La medida de la posición del pico de difracción para 39 orientaciones diferentes permite resolver el sistema de ecuaciones del que se obtienen el tensor de tensiones y los tensores gradientes de tensiones en dirección longitudinal, transversal y en profundidad (711). Este cálculo se ha llevado a cabo aplicando un software comercial (PC-Stress, versión 2.61) desarrollado por Philips.

\section{RESULTADOS Y DISCUSIÓN.}

A continuación se presentan, muy brevemente, los resultados más representativos, tanto de tensiones residuales macroscópicas, como de microestructura y evolución de la anchura de los picos, relacionado esto último con las tensiones microscópicas.

\subsection{Tensiones residuales macroscópicas.}

En las figuras 1a y $1 \mathrm{~b}$ se presentan respectivamente la evolución en profundidad de las tensiones tangencial $\left(\sigma_{11}\right.$ dirección de corte) y radial $\left(\sigma_{22}\right.$ dirección de avance de herramienta) en los tres casos de torneado duro estudiados: muestra sin mecanizar, mecanizada con herramienta nueva y mecanizada con herramienta usada. Se ha observado que en el interior del material, lejos de la superficie, el torneado
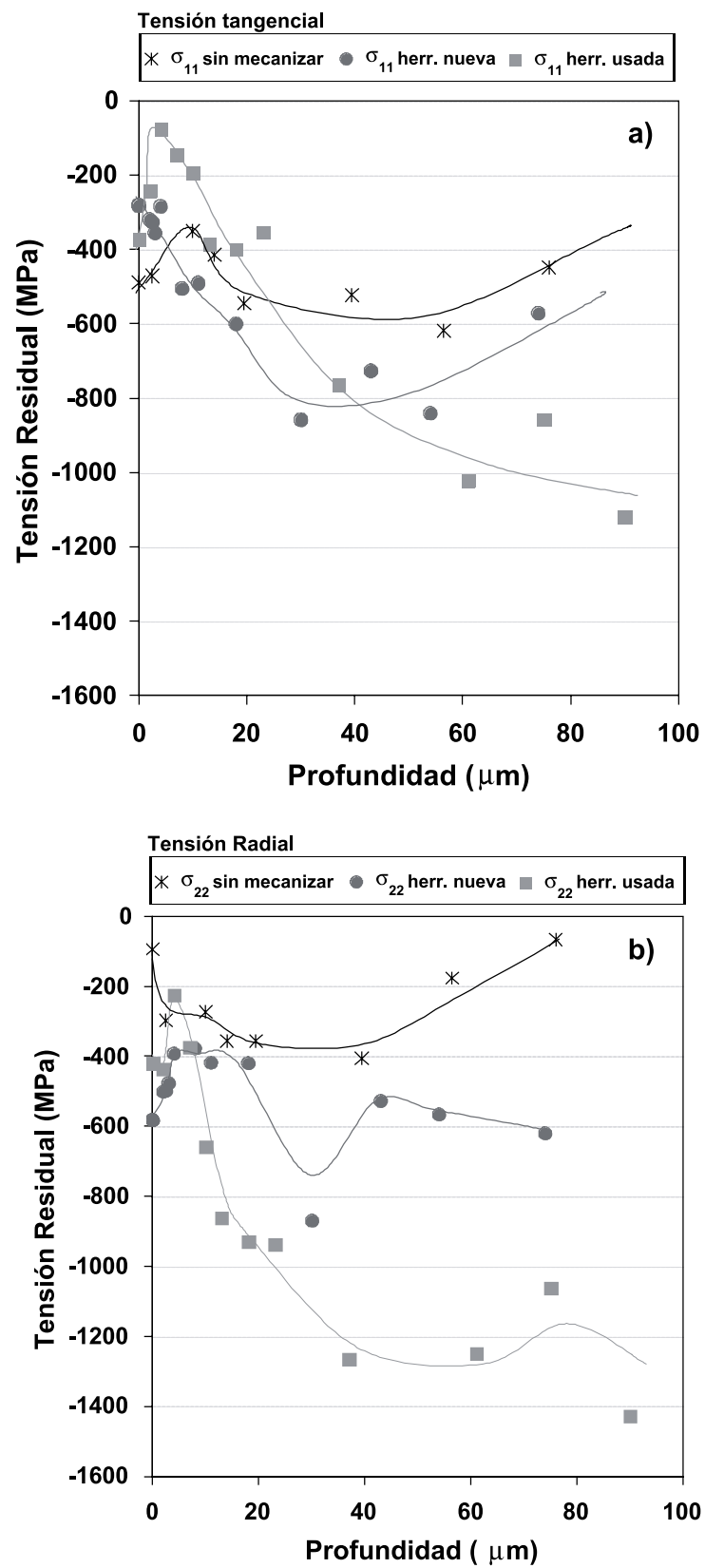

Figura 1 Distribución de tensiones residuales en el acero de herramienta F-521 tras mecanizado por torneado duro: a) $\sigma_{11}$, tensión tangencial y b) $\sigma_{22}$, tensión radial. 
duro genera tensiones residuales más compresivas que las presentes en la muestra sin mecanizar. Asimismo, para espesores superiores a 20 micras se observa una fluctuación de los valores de tensiones obtenidos. Estas fluctuaciones son debidas a la dificultad en obtener una buena calidad superficial tras el proceso de electropulido en este material bifásico. El torneado con herramienta desgastada produce una alteración mayor en el estado de tensiones, ya que provoca una modificación de la microestructura del material hasta profundidades mayores, como se observa en las figuras 2 y 3 . En el interior del material mecanizado se obtienen tensiones más compresivas, lo que indica que en esa zona el efecto dominante es el mecánico (12). En la zona próxima a la superficie el efecto dominante es el térmico y se observa que cuanto mayor es el desgaste de la herramienta (mayor generación de calor por fricción) las tensiones generadas son menos compresivas, y por tanto más perjudiciales, ya que en esa zona la formación de grietas es más probable.

Del estudio de las probetas sometidas a distintas fuentes de rectificado se observa que, dependiendo del proceso utilizado, pueden aparecer tensiones tractivas (12) en la zona cercana a la superficie (figura 4), que pueden favorecer el inicio y propagación de grietas. Esto indica la relevancia de la elección de unos parámetros de mecanizado adecuados con objeto de garantizar un buen comportamiento y larga vida en servicio de las piezas sometidas a dichos procesos de mecanizado. Para los dos materiales estudiados (acero inoxidable 17/7 PH y Ti-6Al-4V) se han obtenido similares resultados.

\subsection{Anchura de los picos y microestructura.}

La variación de la anchura del pico es simultánea a la deformación plástica y caracteriza las microtensiones, que están directamente relacionadas con la microestructura (8). El estudio de la anchura de los picos da únicamente una información cualitativa, no cuantitativa, de la evolución de las microtensiones. Es por ello que en el presente trabajo no se presentan valores absolutos de las microtensiones existentes en el material.

La muestra sometida a torneado duro con herramienta gastada es la que ha sufrido mayores cambios microestructurales (aparición de capa blanca y rotura de los carburos alargados formados en la laminación, figura 3) (11). Este hecho se ve reflejado también en la evolución de la anchura de los picos representada en la figura 2. El torneado con herramienta gastada genera altas temperaturas en el material. La superficie se enfría más rápidamente dando lugar a una capa superficial amorfa o nanocristalina (capa blanca, de unas 2 micras de espesor) con gran distorsión de red, lo que se corresponde con los altos valores de la anchura de los picos, debidos tanto a dicha capa blanca como a la alta deformación plástica que tiene lugar en las 10 primeras micras de profundidad. El interior se enfría más lentamente, dando lugar a restauración y formación de martensita sobrerevenida, obteniéndose una densidad de dislocaciones menor que la presente en el material sin mecanizar, lo que concuerda perfectamente con lo observado: la anchura de los picos en el interior del material mecanizado con herramienta desgastada es menor que en el caso del material sin mecanizar (11).

Por otra parte el torneado con herramienta nueva no genera temperaturas tan elevadas en el material y da lugar a una restauración parcial con la consiguiente formación de una capa de martensita sobrerevenida en la superficie, que se corresponde con los valores de anchura de pico menores que en el caso del material sin mecanizar. El interior del material apenas se ve afectado en el caso de torneado con herramienta nueva, lo que concuerda con el hecho de que la anchura de los picos en el interior del material alcanza el valor del material no mecanizado (11).

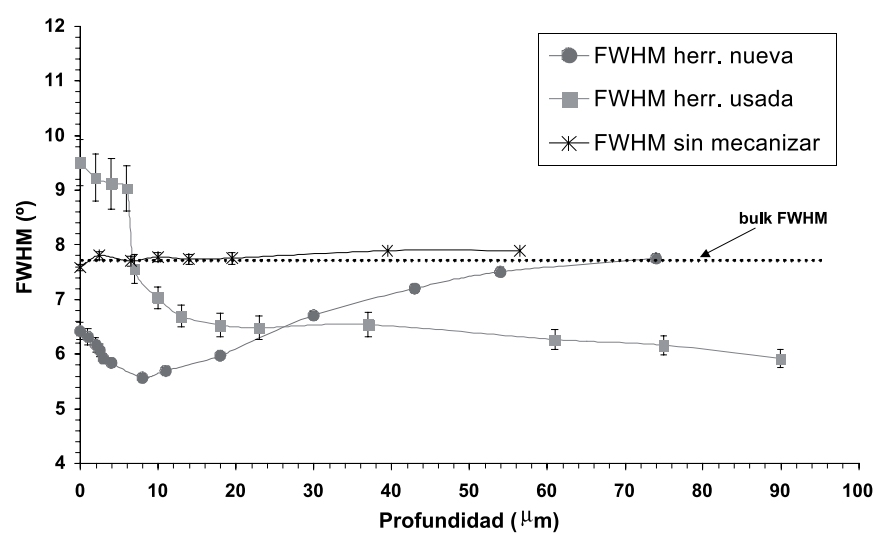

Figura 2. Evolución de la anchura del pico con la profundidad en acero F-521 sometido a torneado.

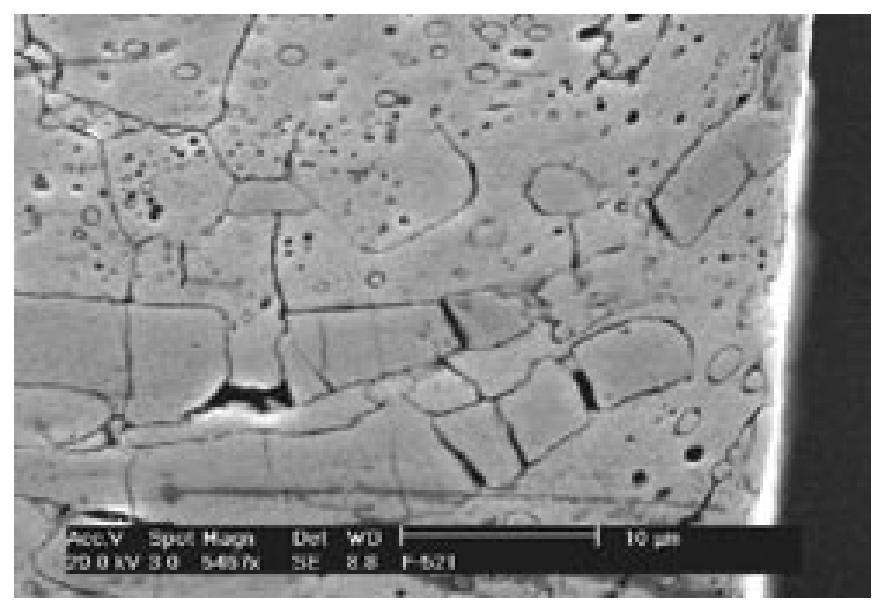

Figura 3. Micrografía obtenida en SEM del acero F-521 (torneado duro) tras ser atacado con Nital.

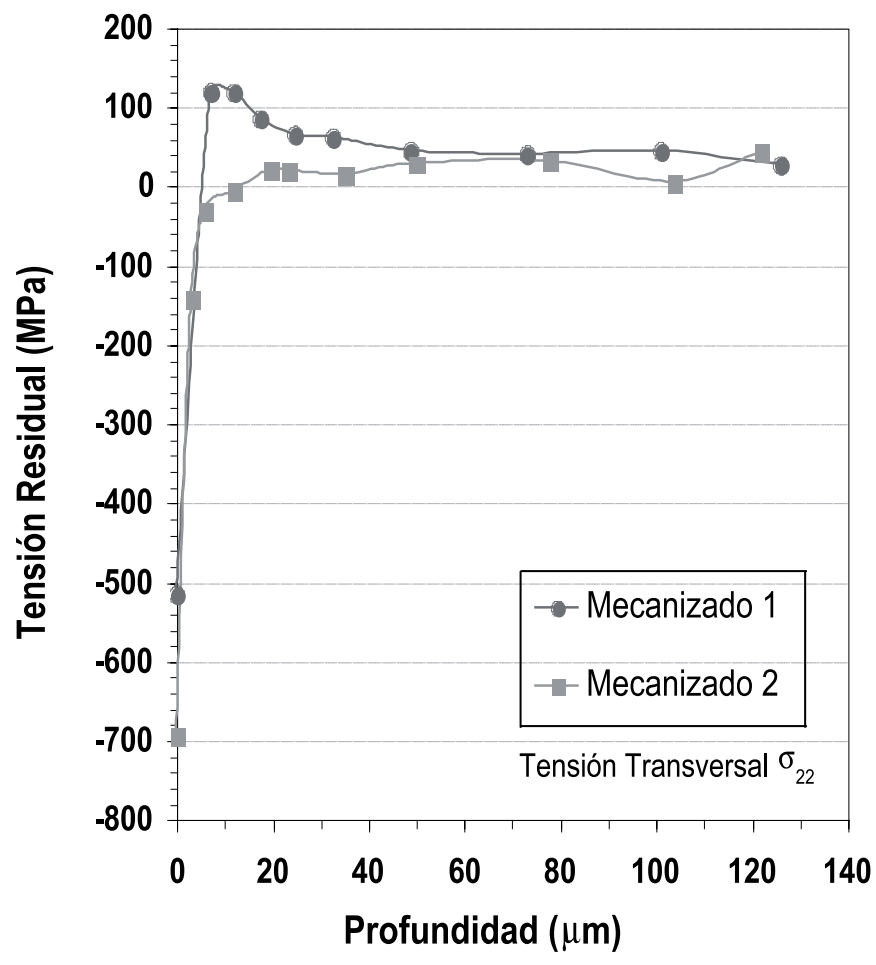

Figura 4. Tensión residual transversal en acero 17/ 7 PH sometido a dos procesos de rectificado diferentes. 
En el caso de probetas sometidas a rectificado se ha observado (figura 5) que la anchura del pico va disminuyendo con la profundidad hasta alcanzar un estado de equilibrio a partir de una determinada profundidad, que se corresponde con la profundidad en la cual se observa un mayor cambio microestructural. En las primeras 8 micras los elevados valores de la anchura de los picos indican la posible presencia de capa blanca así como la alta deformación plástica generada por el proceso de rectificado (similar a lo ocurrido por torneado con herramienta gastada). En el interior del material se alcanzan los valores de anchura de pico del material no mecanizado, al igual que lo observado en el caso de torneado con herramienta nueva.

\section{CONCLUSIONES.}

El torneado duro induce tensiones residuales en el interior del material más compresivas que las obtenidas mediante rectificado. Esto hace que hoy en día se vislumbre el torneado duro como una alternativa interesante al rectificado. No obstante, la elección de uno u otro proceso, así como de los parámetros a aplicar en cada caso, debe basarse también en otros aspectos, como calidad superficial de la pieza final, tiempo de mecanizado (productividad),...

La temperatura inducida por fricción entre pieza y herramienta aumenta con el desgaste de la herramienta, lo que resulta en una disminución de las tensiones residuales compresivas inmediatamente debajo de la superficie. El uso de herramienta desgastada es por tanto más perjudicial para la calidad superficial final de la pieza. En el interior el efecto mecánico de presión es dominante al de temperatura, y se obtienen tensiones residuales más compresivas que las presentes en la muestra sin mecanizar, siendo estas tensiones tanto más compresivas cuanto mayor es el desgaste de la herramienta. Esto establece la importancia de evaluar el estado de tensiones no sólo en la superficie, sino también en el interior del material mecanizado. De este modo, puede determinarse cuál es el proceso de mecanizado óptimo a aplicar.

\section{BIBLIOGRAFÍA}

1. A. M. Abrao, D. K. Aspinwall. "The surface integrity of turned and ground hardened bearing steel". Wear, 196 (1996) 279-284.

2. W. König, R. Komanduri, H. K. Tönshoff and G Ackershott. "Machining of Hard Materials". Annals of the CIRP. Vol. 33/2/1984.
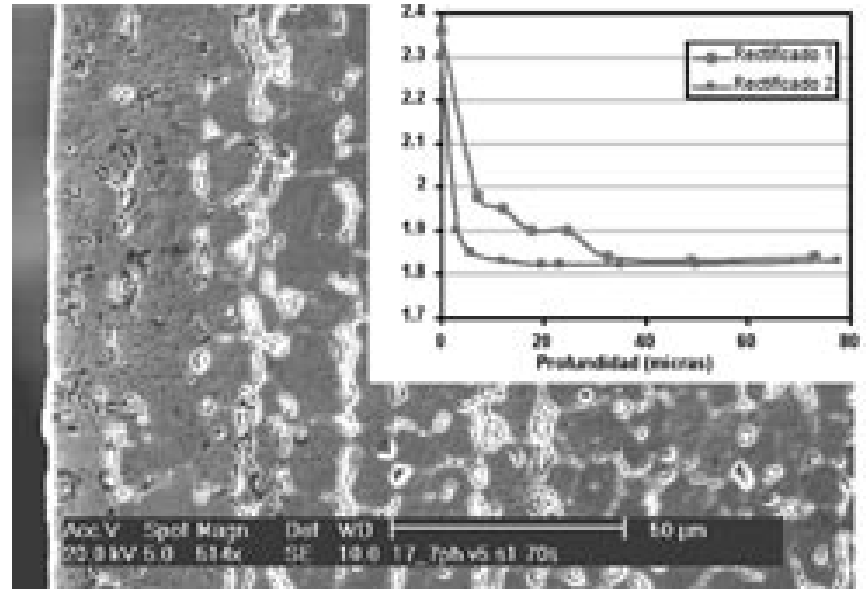

Figura 5. Micrografía obtenida en SEM del acero 17 / 7PH (rectificado) tras ser atacado con ácido oxálico y evolución de la anchura de los picos con la profundidad.

3. W. König, A. Berktold, K. F. Koch. "Turning grinding. a comparison of surface integrity aspects and attainable accuracies". Annals of the CIRP. Vol. 42/1/1993.

4. K. Nealey. "Surface integrity of machined components. Microstructural aspects". Metals. Mater. (1988) 93-96.

5. M. C. Shaw. Principles of Abrasive Processing, 1996, Oxford University Press, New York, Chapter 9-10. P 213-313.

6. M. G. Moore and W. P. Evans. "Mathematical correction for stress in removed layers in X-Ray diffraction residual stress analysis". SAE Transactions. Vol. 66, 1958. P 340-345.

7. I. C. Noyan and J. B. Cohen. Residual stress. measurement by diffraction and interpretation. Ed. Springer-Verlag, 1987.

8. Handbook of measurement of residual stresses. Edited by Jian Lu. Society for Experimental Mechanics, Ed. The Fairmont Press, Inc, 1996.

9. H. Wern. "Influence of measurement and evaluation parameters on stress distributions investigated by X-rays". Strain, November 1991 127-136.

10. A. Peiter and H. Wern. "Simultaneous X-ray measurements in-situ of triaxial stresses, Poisson's ratio and the stress free lattice spacing". Strain, August 1987 103-107.

11. N.Ordás, M.L.Penalva, J.Fernández, C. García-Rosales "Residual stresses in tool steel due to hard-turning" (To be published in Journal of Applied Crystallography)

12. P. Vomacka and H. Walburger. "Residual stresses due to hard-machining. industrial experiences". 5th European Conference on Residual Stresses. Proceedings ECRS5. Materials Science Forum, Vols. 347-349 (2000) pp. 592-597. Trans Tech Publications, Switzerland.

Recibido: 1.2 .03

Aceptado: 30.11 .03 